\title{
Electronic States and Luminescence in Porous Silicon Quantum Dots: The Role of Oxygen
}

\author{
M. V. Wolkin, J. Jorne, * and P. M. Fauchet ${ }^{\dagger}$ \\ Materials Science Program, University of Rochester, Rochester, New York 14627 \\ G. Allan and C. Delerue \\ Département Institut Supérieur d'Electronique du Nord, Institut d'Electronique et de Microélectronique du Nord, \\ 41 boulevard Vauban 59046 Lille cédex, France
}

(Received 25 September 1998)

\begin{abstract}
Depending on the size, the photoluminescence (PL) of silicon quantum dots present in porous silicon can be tuned from the near infrared to the ultraviolet when the surface is passivated with Si-H bonds. After exposure to oxygen, the PL shifts to the red by as much as $1 \mathrm{eV}$. This shift and the changes in PL intensity and decay time, show that both quantum confinement and surface passivation determine the electronic states of silicon quantum dots. A theoretical model in which new electronic states appear in the band gap of the smaller quantum dots when a $\mathrm{Si}=\mathrm{O}$ bond is formed, is in good agreement with experiments. This result clarifies the controversy regarding the PL mechanisms in porous silicon. [S0031-9007(98)08118-6]

PACS numbers: 78.55.Mb, 71.24. $+\mathrm{q}$
\end{abstract}

The study of silicon quantum dots is a very active field of research, because of the interesting fundamental physical properties of these mesoscale objects and of promising applications in advanced electronic devices [1] and optoelectronic devices [2]. Of all the forms of silicon containing quantum dots, porous silicon (PSi) is the one that has attracted the most attention to date [3-5], mostly because of its intense visible photoluminescence (PL). Numerous models have been proposed to explain its PL, including quantum confinement [6-8], surface states [9], defects in the oxide [10], and even specific chemical species (e.g., siloxenes [11]). Presently, although a detailed understanding of the PL has yet to be achieved [4], it is usually accepted that the band gap opens as a result of quantum confinement, which pushes the PL in the visible for crystallite sizes below $5 \mathrm{~nm}$ [8]. It has even been proposed that quantum confinement provides an explanation for all the slow PL from PSi [3]. However, many groups have reported that when the crystallite size decreases to a few nanometers, the PL in air does not increase much beyond $2.1 \mathrm{eV}$ even when the crystallite size drops well below $3 \mathrm{~nm}[12,13]$. This observation does not coincide with theory, which predicts a much larger opening of the band gap, in excess of $3 \mathrm{eV}$ for sizes below $2 \mathrm{~nm}[8,14]$.

The goal in this Letter is to solve that apparent contradiction and to give a global explanation for the visible PL and the electronic states in PSi. We examine oxygen-free PSi samples with different porosities and emitting throughout the visible spectrum (red to blue, following Ref. [15]). After exposure to air, a redshift of the PL is observed, which can be as large as $1 \mathrm{eV}$ for blue luminescent samples that contain crystallites smaller than $2 \mathrm{~nm}$. After a full characterization of the PL properties and the chemical composition, we propose a model that describes the recombination of carriers over a wide range of nanocrystal sizes and different environments. In this model, the redshift of the PL immediately after exposure to oxygen is related to the trapping of an electron (or even an exciton) by $\mathrm{Si}=\mathrm{O}$ bonds that produce localized levels in the bandgap of nanocrystals smaller than $\sim 3 \mathrm{~nm}$.

The PSi samples were formed by electrochemical etching followed by photoassisted stain etching of $6 \Omega \mathrm{cm}$ $p$-type $\mathrm{Si}$ wafers at current densities of $8-50 \mathrm{~mA} / \mathrm{cm}^{2}$ using 10\%-25\% HF:ethanol solutions. Stain etching, accomplished under illumination with a $500 \mathrm{~W}$ halogen lamp, was used to further increase the porosity [16]. All samples were rinsed in ethanol and immediately transferred into an Ar environment. Special care was taken that the samples never be exposed to air. The blue sample was the only one measured under vacuum. A second set of identical samples were rinsed in ethanol and then exposed to air.

The PL spectra were obtained at room temperature by using either a pulsed excitation nitrogen laser $(337 \mathrm{~nm})$ or a continuous excitation $\mathrm{HeCd}$ laser $(325 \mathrm{~nm})$. Figure 1a shows the PL spectra of five types of oxygen-free PSi samples with different porosities. Stable red, orange, yellow, green, and blue spectra, in increasing order of porosity, were obtained and measured when the samples were kept under the Ar environment. The PL intensity increased by several orders of magnitude as the PL wavelength changed from the red to the yellow, consistent with the quantum confinement model. However, as the porosities were further increased, the PL intensity dropped, at least in part due to the fact that the penetration depth became larger than the PSi thickness. Figure 1b shows how the spectra were modified after the samples had been exposed to air for $24 \mathrm{~h}$. Two major trends were observed. The PL from the samples emitted in the blue to orange region was redshifted, and the PL intensities decreased. The magnitude of the redshift increased with increasing porosities, and the PL peak energy eventually 
saturated near $2.1 \mathrm{eV}(590 \mathrm{~nm})$ for the green and blue samples. For the samples that initially emitted in the red, no redshift was observed, and the PL remained the same whether the samples were stored in Ar or aged in air.

We measured the PL decay time in Ar and in air at the maximum PL energy of each sample, and found it be in the microsecond range. The lifetimes were obtained by fitting a stretched exponential function to the experimental decay transients. In Ar, as the porosity increased, the lifetime decreased monotonically, from $32 \mu \mathrm{s}$ (in the red) to $0.07 \mu \mathrm{s}$ (in the blue). After exposure to air, the lifetimes also decreased with increasing porosities, until they reached $2 \mu$ s (initially green PL), after which they stayed constant. In addition, the difference between the lifetime measured in air and Ar increased with increasing porosity.

In order to understand the origin of the redshift after $24 \mathrm{~h}$ of exposure to air, we investigated the PL in different gas environments. A large redshift was observed as soon as the samples were transferred from Ar to a pure oxygen atmosphere. In contrast, no redshift at all was detected when the samples were kept in pure hydrogen or in vacuum. We hypothesized that the large redshift was related to surface passivation, and probably the presence of oxygen.

To test this hypothesis, we investigated the evolution of the chemical coverage of an Ar-stored sample as it was exposed to air. Figure $2 \mathrm{a}$ shows the evolution of the Fourier transform infrared spectroscopy (FTIR) transmission spectra of a blue-green sample before $(t=$ $0)$ and after exposure to air $(t>0)$. The spectrum of the fresh sample showed strong absorption bands near 2100 and $664 \mathrm{~cm}^{-1}$, associated with the stretching and deformation of $\mathrm{Si}-\mathrm{H}_{n}(n=1-3)$, and no sign of an oxygen peak, which confirmed that the samples stored in Ar were well passivated by hydrogen and free of oxygen.
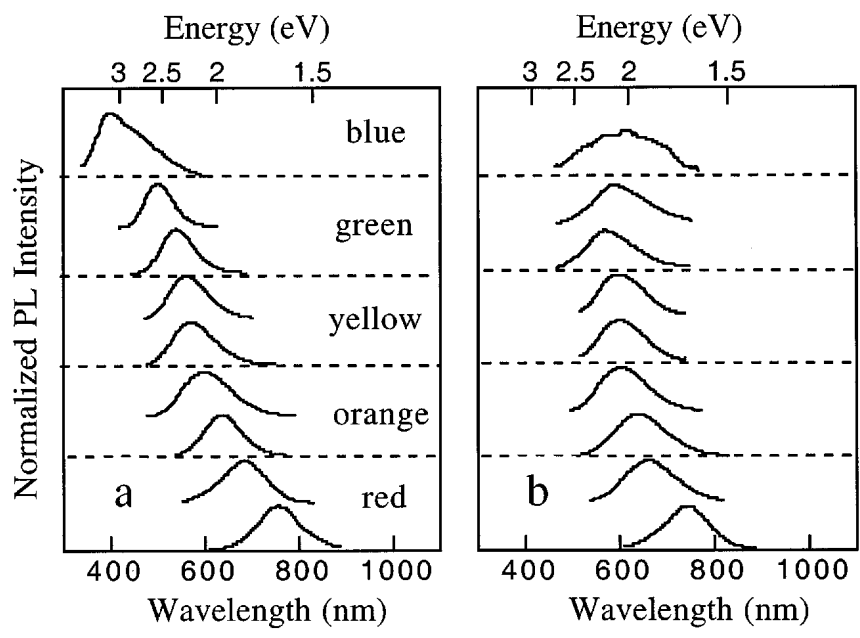

FIG. 1. Room temperature photoluminescence spectra from PSi samples with different porosities kept under Ar atmosphere (a) and after exposure to air (b).
As fast as $3 \mathrm{~min}$ after exposure to air, a $\mathrm{Si}-\mathrm{O}-\mathrm{Si}$ feature at $1070 \mathrm{~cm}^{-1}$ appeared and gradually became dominant. In addition, after $100 \mathrm{~min}$, a new peak was observed at $850 \mathrm{~cm}^{-1}$ related to $\mathrm{Si}-\mathrm{O}-\mathrm{H}$. The $\mathrm{Si}-\mathrm{H}_{n}$ peaks at $2100 \mathrm{~cm}^{-1}$ decreased progressively with time and disappeared after $24 \mathrm{~h}$. When the samples were exposed to air for longer than $200 \mathrm{~min}$, no significant change in the $\mathrm{Si}-\mathrm{O}-\mathrm{Si}$ and $\mathrm{Si}-\mathrm{O}-\mathrm{H}$ peaks was observed, indicating stabilization of the surface chemical coverage.

As the surface passivation was gradually changing, the PL was redshifted. Figure $2 b$ shows the progressive redshift of the PL with time. Most of it was obtained in the first few minutes of exposure, and stabilization was achieved after aging for $200 \mathrm{~min}$. This result correlates well with the change of the surface passivation. Based on the previous results, we argue that both porosity (or size) and chemical coverage dictate the recombination mechanism.

All these results suggest that the electron-hole recombination in samples exposed to oxygen occurs via carriers trapped in oxygen-related localized states that are stabilized by the widening of the gap induced by quantum confinement. Thus we have applied electronic structure calculations to various situations involving oxygen atoms at the surface of Si clusters. As expected because of the large offset between bulk $\mathrm{SiO}_{2}$ and $\mathrm{Si}(\sim 4 \mathrm{eV})$, for normal $\mathrm{Si}-\mathrm{O}-\mathrm{Si}$ bonds, we do not find any localized gap state. Similar results are obtained for $\mathrm{Si}-\mathrm{O}-\mathrm{H}$ bonds. But, when nanocrystalline $\mathrm{Si}$ is oxidized and a $\mathrm{Si}-\mathrm{O}-\mathrm{Si}$
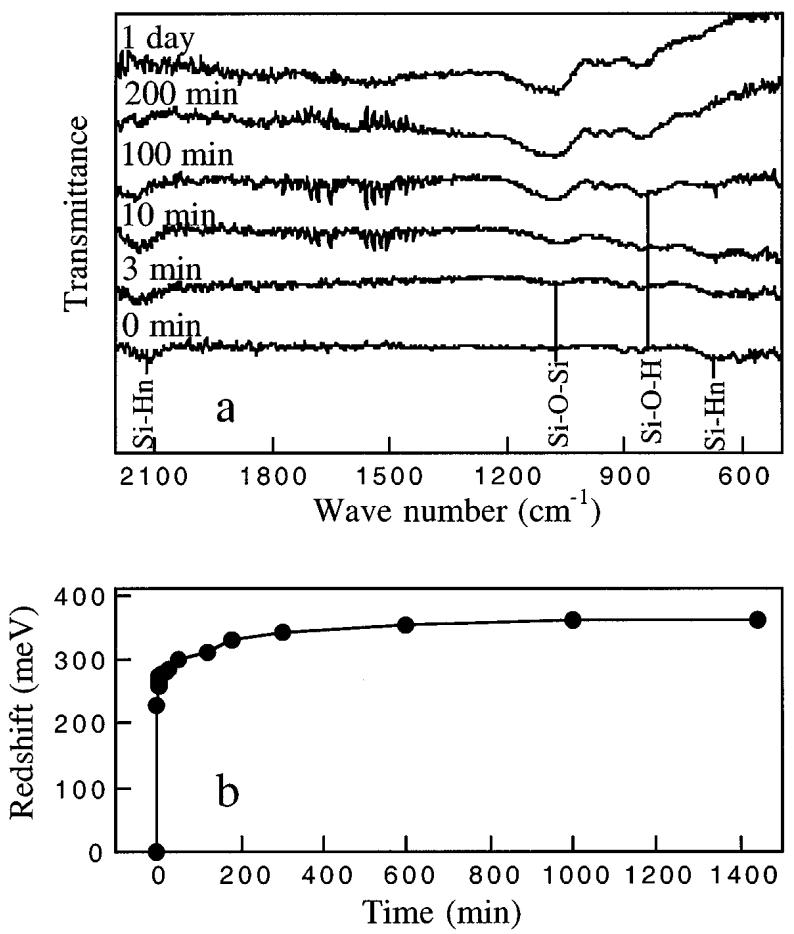

FIG. 2. Evolution of the FTIR transmission spectra (a) and the PL redshift (b), for a blue-green sample as a function of air exposure time. 
layer is formed on the surface, the $\mathrm{Si}-\mathrm{Si}$ or $\mathrm{Si}-\mathrm{O}-\mathrm{Si}$ bonds are likely to weaken or break in many places because of the large stress at the $\mathrm{Si} / \mathrm{SiO}_{2}$ interface [17]. Some mechanisms can act to passivate the dangling bonds [18]. $\mathrm{A} \mathrm{Si}=\mathrm{O}$ double bond is more likely to be formed and stabilize the interface, since it requires neither a large deformation energy nor an excess element. It would also terminate two dangling bonds. Such bonds have been suggested at the $\mathrm{Si} / \mathrm{SiO}_{2}$ interface [18].

The electronic structure of $\mathrm{Si}$ clusters with one $\mathrm{Si}=\mathrm{O}$ bond (the other dangling bonds being saturated by hydrogen atoms) is calculated as a function of the cluster size. We use a self-consistent tight-binding method closely following Ref. [19]. To correctly reproduce the valence and conduction bands of bulk silicon, we use the parameters of Ref. [20], which include three-center integrals up to the third nearest neighbors. The interactions between $\mathrm{Si}$ and $\mathrm{O}$ are obtained from Ref. [21]. The Hamiltonian matrix is diagonalized using an inverted Lanczos iteration procedure which allows us to treat clusters up to $\sim 5 \mathrm{~nm}$. The optical matrix elements are obtained as in Ref. [8], i.e., without the assistance of phonons. For small clusters we have verified the consistency of our results with those of $a b$ initio local density calculations performed with the code of Ref. [22]: Similar electronic structures are obtained even if the positions of the states in the gap cannot be compared exactly because of the wellknown underestimation of the band gap by local density calculations.

The calculated electronic states in Si nanocrystals are presented in Fig. 3. The model suggests that when a $\mathrm{Si}$ cluster is passivated by hydrogen, recombination is via free excitons states for all sizes. The PL energy is equal to the free exciton band gap and follows the quantum confinement model. However, if the Si nanocrystallite is passivated by oxygen, a stabilized electronic state (or even a trapped exciton) may be formed on the $\mathrm{Si}=\mathrm{O}$ covalent bond. The electron state is a $p$ state, localized on the $\mathrm{Si}$ atom, and the hole size is a $p$ state, localized on the oxygen atom. For oxygen-passivated clusters, three different recombination mechanisms are suggested, depending on the size of the cluster. Each zone in Fig. 3 corresponds to a different mechanism. In zone I, recombination is via free excitons. As the cluster size decreases, the PL energy increases, exactly as predicted by quantum confinement. There is no redshift whether the surface termination is hydrogen or oxygen, since the band gap is not wide enough to stabilize the $\mathrm{Si}=\mathrm{O}$ surface state. In zone II, recombination involves a trapped electron and a free hole. As the size decreases, the PL emission energy still increases, but not as fast as predicted by quantum confinement, since the trapped electron state energy is size independent. In zone III, recombination is via trapped excitons. As the size decreases, the PL energy stays constant, and there is a large PL redshift when the nanocrystallite surface becomes exposed to oxygen.

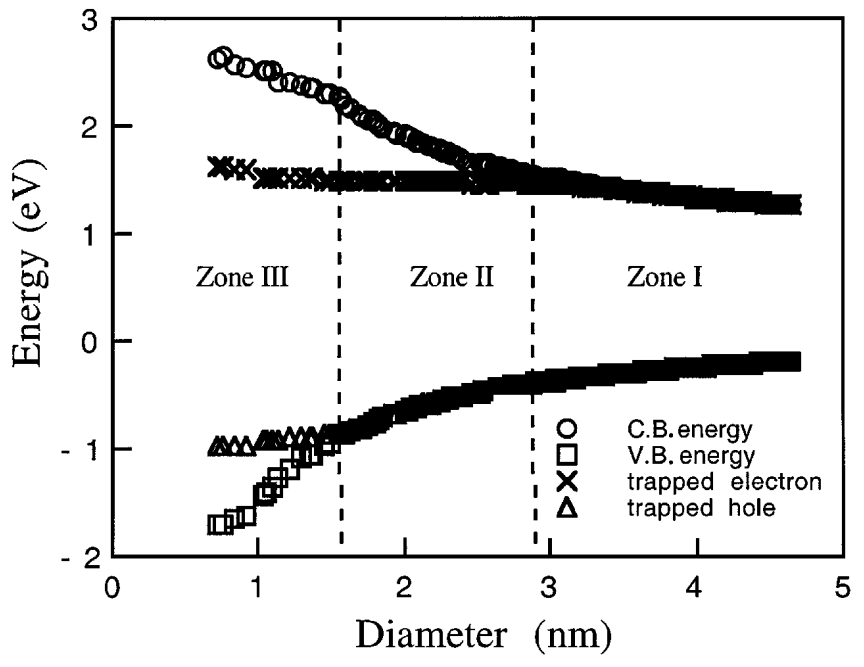

FIG. 3. Electronic states in Si nanocrystals as a function of cluster size and surface passivation. The trapped electron state is a $p$-state localized on the $\mathrm{Si}$ atom of the $\mathrm{Si}=\mathrm{O}$ bond and the trapped hole state is a $p$-state localized on the oxygen atom.

In order to compare the calculations with experimental results, it is necessary to evaluate the nanocrystallite size. In ultrahigh porosity samples, the crystallites are very small $(\leq 2 \mathrm{~nm})$, and there is no obvious way to measure their size reliably. If we accept that the PL in PSi stored under an Ar atmosphere is due to recombination via free excitonic states, the PL energy itself can be used to deduce the average size. Therefore, we have equated the calculated excitonic band gap and the peak PL energy to obtain the size of the nanocrystals in each PSi sample. Figure 4 presents the experimental PL energy (measured in Ar and air), and the calculated PL energy (free exciton energy and lowest transition energy for a nanocrystal with a $\mathrm{Si}=\mathrm{O}$ bond) as a function of nanocrystal sizes. The agreement between experiments and theory is good, despite the simplicity of the model. As expected, three zones are observed; zone I for free excitonic recombination, independent of the surface bonds; zone II where, in the presence of a $\mathrm{Si}=\mathrm{O}$ bond, the electron is localized on that bond but the hole is free; and zone III where the electron and the hole are trapped on the $\mathrm{Si}=\mathrm{O}$ bond ("trapped exciton"). The magnitude of the measured redshift is as calculated in the model. In addition, we note that the experimental and theoretical PL decay lifetimes are in agreement. Therefore, we conclude that the model proposed for the electronic states and the luminescence of porous silicon quantum dots explains the experimental data.

Taken together, the results in this paper indicate that: (a) The band gap of $\mathrm{Si}$ nanocrystals opens by quantum confinement. The emission energy increases with decreasing sizes, covering the entire visible spectrum; (b) surface passivation plays an important role, especially 


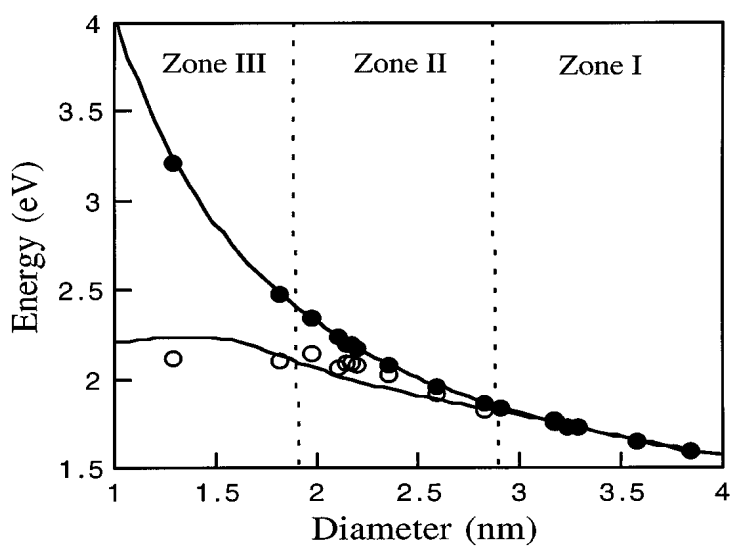

FIG. 4. Comparison between experimental and theoretical PL energies as a function of crystallite size. The upper line is the free exciton band gap and the lower line is the lowest transition energy in the presence of a $\mathrm{Si}=\mathrm{O}$ bond. The and $\bigcirc$ are the peak PL energies obtained from Figs. 1a and $1 \mathrm{~b}$, respectively. In zone I the PL peak energies are identical, whether the samples have been exposed to oxygen or not.

at small sizes $(d<3 \mathrm{~nm})$. The recombination mechanism in oxidized nanocrystallites of this size is different from that in hydrogen passivated crystallites; (c) the redshift upon oxidation is related to recombination involving a trapped electron or exciton. It explains the huge Stokes shift observed for the smallest nanocrystallites and the upper limit of the emission energy $(2.1 \mathrm{eV})$, which is independent of size. The agreement between the theoretical model and the experimental results indicates that it is likely that the carriers are trapped in the $\mathrm{Si}=\mathrm{O}$ bond. The fact that a redshift was observed in a pure oxygen environment shows that other bonds (such as $\mathrm{Si}-\mathrm{O}-\mathrm{H}$ ) are less likely to be responsible for the PL; (d) the nanocrystal sizes in the PSi samples investigated by most researchers are larger than $3 \mathrm{~nm}$ and display red or orange emission. In those, quantum confinement alone can explain most of the results, and surface passivation has a negligible impact on the radiative recombination mechanism; (e) many researchers define "fresh samples" as samples which have been exposed to air from a few minutes up to half an hour. However, our results show that oxidation occurs in seconds, changing the sample's recombination mechanism and optical properties. Our results also suggest that an oxide may not provide a good passivation in small $\mathrm{Si}$ crystallites, in contrast to bulk $\mathrm{Si}$. This observation may be very important for future nanoelectronics applications.

Support from the U.S. Army Research Office and the Electric Power Research Institute is gratefully ac- knowledged. We thank L. Tsybeskov, C. Wamsley, and G. Wicks for technical assistance. The "Institut d'Electronique et de Microélectronique du Nord" is "Unite mixte 9929 du Centre National de la Recherche Scientifique."

*Also with Department of Chemical Engineering.

${ }^{\dagger}$ Also with Department of Electrical and Computer Engineering.

[1] A. Fowler, Phys. Today 50, No. 10, 50 (1997).

[2] K. D. Hirschman, L. Tsybeskov, S. P. Duttagupta, and P. M. Fauchet, Nature (London) 384, 338 (1996).

[3] A. G. Cullis, L. T. Canham, and P. D. J. Calcott, J. Appl. Phys. 82, 909 (1997).

[4] P. M. Fauchet, J. Lumin. 70, 294 (1996).

[5] R. T. Collins, P. M. Fauchet, and M. A. Tischler, Phys. Today 50, No. 1, 24 (1997).

[6] L. T. Canham, Appl. Phys. Lett. 57, 1046 (1990).

[7] V. Lehman and U. Gösele, Appl. Phys. Lett. 58, 865 (1991).

[8] J. P. Proot, C. Delerue, and G. Allan, Appl. Phys. Lett. 61, 1948 (1992).

[9] F. Koch, V. Petrova-Koch, and T. Muschik, J. Lumin. 57, 271 (1993).

[10] S. M. Prokes, Appl. Phys. Lett. 62, 3244 (1993).

[11] M.S. Brandt, H. D. Fuchs, M. Stutzmann, J. Weber, and M. Cardona, Solid State Commun. 81, 307 (1992).

[12] J. von Behren, T. Van Buuren, M. Zacharias, E. H. Chimowitz, and P. M. Fauchet, Solid State Commun. 105, 317 (1998).

[13] S. Schuppler et al., Phys. Rev. B 52, 4910 (1995).

[14] L. Wang and A. Zunger, Phys. Chem. 98, 2158 (1994).

[15] H. Mizuno, H. Koyama, and N. Koshida, Appl. Phys. Lett. 69, 3779 (1996).

[16] H. Koyama and N. Koshida, J. Appl. Phys. 74, 6365 (1993).

[17] A. Ourmazd, D. W. Taylor, J. A. Rentschler, and J. Bevk, Phys. Rev. Lett. 59, 213 (1987).

[18] F. Herman and R. V. Kasowski, J. Vac. Sci. Technol. 19, 395 (1981).

[19] M. Lannoo and J. Bourgoin, in Point Defects in Semiconductors I, edited by M. Cardona (Springer-Verlag, New York, 1981).

[20] C. Tserbak, H. M. Polatoglou, and G. Theodorou, Phys. Rev. B 47, 7104 (1993).

[21] M. O'Reilly and J. Robertson, Phys. Rev. B 27, 3780 (1983).

[22] DMol User Guide (Molecular Simulations, San Diego, 1996). 